University of Nebraska - Lincoln

DigitalCommons@University of Nebraska - Lincoln

Identification and Quantification of 11 Airborne Biochemicals Emitted by the Brown Recluse and Another Primitive Hunting Spider Using Headspace Solid-Phase Microextraction-GC/MS

Zachary Foulks

Jennifer Parks

William Stoecker

Charles Kristensen

Eileen A. Hebets

See next page for additional authors

Follow this and additional works at: https://digitalcommons.unl.edu/bioscihebets

Part of the Animal Sciences Commons, Behavior and Ethology Commons, Biology Commons, Entomology Commons, and the Genetics and Genomics Commons

This Article is brought to you for free and open access by the Papers in the Biological Sciences at DigitalCommons@University of Nebraska - Lincoln. It has been accepted for inclusion in Eileen Hebets Publications by an authorized administrator of DigitalCommons@University of Nebraska - Lincoln. 
Authors

Zachary Foulks, Jennifer Parks, William Stoecker, Charles Kristensen, Eileen A. Hebets, and Honglan Shi 
Published in Analytical and Bioanalytical Chemistry 413:26 (2021), pp. 6605-6615; doi: 10.1007/s00216021-03626-8

Copyright (C) 2021 Springer-Verlag GmbH Germany, part of Springer Nature. Used by permission. Submitted July 12, 2021; revised August 17, 2021; accepted August 18, 2021; published online September 2, 2021.

Supplemental material for this article is available following the references.

\title{
Identification and Quantification of 11 Airborne Biochemicals Emitted by the Brown Recluse and Another Primitive Hunting Spider Using Headspace Solid-Phase Microextraction-GC/MS
}

\author{
Zachary Foulks, ${ }^{1}$ Jennifer Parks, ${ }^{1,2}$ William Stoecker, ${ }^{2}$ Charles Kristensen, ${ }^{3}$ \\ Eileen A. Hebets, ${ }^{4}$ and Honglan Shi ${ }^{1}$
}

1. Department of Chemistry, Missouri University of Science and Technology, Rolla, Missouri, USA

2. The Dermatology Center and SpiderTek, Rolla, MO 65401, USA

3. SpiderPharm, Yarnell, Arizona, USA

4. School of Biological Sciences, University of Nebraska-Lincoln, Lincoln, Nebraska, USA

Corresponding author - Honglan Shi, Department of Chemistry, Missouri University of Science and Technology, 400 W 11th Street, Rolla, MO, 65409, USA, email honglan@mst.edu

\begin{abstract}
Loxosceles reclusa, or brown recluse spider, is a harmful household spider whose habitat extends throughout the Midwest in the USA and other regions in the world. The pheromones and other biomolecules that facilitate signaling for brown recluses and other spider species are poorly understood. A rapid and sensitive method is needed to analyze airborne spider signaling biomolecules to better understand the structure and function of these biochemicals in order to control the population of the spiders. In this study, we developed a novel headspace solid-phase microextraction (HS-SPME)GC/MS method to analyze potential pheromones and biomolecules emitted by the brown recluse
\end{abstract}


spider. The method is highly selective and sensitive for biomolecule identification and quantification from a single live spider. Using this novel nondestructive HS-SPME-GC/MS technique, we identified 11 airborne biomolecules, including 4-methylquinazoline, dimethyl sulfone, 2-methylpropanoic acid, butanoic acid, hexanal, 3-methylbutanoic acid, 2-methylbutanoic acid, 2,4-dimethylbenzaldehyde, 2-phenoxyethanol, and citral (contains both isomers of neral and geranial). Some of these airborne biomolecules were also reported as semiochemicals associated with biological functions of other spiders and insects. The method was also applied to study the airborne biochemicals of Plectreurys tristis, another primitive hunting spider with a poor web, enabling quantitation of the same compounds and demonstrating a difference in signaling molecule concentrations between the two species. This method has potential application in the study of pheromones and biological signaling in other species, which allows for the possibility of utilizing attractant or deterrent functions to limit household populations of harmful species.

Keywords: pheromone, semiochemical, Loxosceles reclusa, Plectreurys tristis, solid-phase microextraction-GC/MS, nondestructive

\section{Introduction}

Recently, researchers have identified a number of compounds with behavioral significance in arachnids [1, 2]. In addition to compounds used for internal metabolic processes and for olfactory signaling purposes among members of the same species, some of the primary areas of study are the pheromones used for attraction and sexual communication. Identification of pheromones and other signaling compounds (collectively termed "semiochemicals") is important both for gaining a greater understanding of their relatively poorly understood metabolic processes and for developing devices better able to capture or otherwise control their populations. If traps are treated with compounds known to attract a particular species at a considerable distance [3], these devices have the potential to more efficiently combat infestations and lower the risk of exposure to harmful and potentially lethal arachnids, such as Atrax robustus (the funnel web spider), Latrodectus mactans (the black widow), and Loxosceles reclusa (the recluse spider). Therefore, the identification of semiochemicals from different species in tandem with behavioral studies has the potential to improve health outcomes for the general population and enable a greater control of insect and arachnid activity. This potential, as yet unexplored, may well be limited by the important role played by other factors in arachnid mating. These other factors include male and female learned sexual experience [4], multimodal signaling employed by some species [5], and silk-based mating behaviors [6].

While a significant number of semiochemicals have been identified for many insect species [7], a comparatively low number of these compounds have been found for arachnids. The first spider semiochemicals identified were the sex pheromones (R)-3-hydroxybutyric acid and its dimer, (R)-3-[(R)-3-hydroxybutyryloxy]-butyric acid, for the spider Linyphia triangularis [8]. This compound was identified through liquid extraction from the web of the spider followed by GC/MS analysis, and it was found to be produced only by adult virgin spiders. Since then, a number of semiochemicals have been identified for various arachnids using a couple of techniques. Liquid extraction from webs followed by GC/MS and/or NMR analysis has remained a popular technique [9-11]. For spider airborne 
compound analysis, researchers have employed a closed loop stripping apparatus [12]. Pheromone effectiveness was then confirmed through behavioral testing. However, these techniques have seen limited success when used with other spider species. The need for destructive sampling in the case of extraction leads to significantly more biological variability between samples and requires a large number of spiders to test. Web sampling has limitations for many species which do not develop enough web to allow effective processing. Extraction of the spider body itself, while possible, is prone to biological variability. Meanwhile, the direct headspace gas collection sampling method currently in use [13] requires disruptive handling of the spider (which could alter chemical production) and is an involved process with relatively low sensitivity. A more effective, efficient, and generally more applicable method is sought for detection of airborne signaling compounds. An ideal method would include little disruption or handling of the spider sample, repeatable sampling of the same spider (so that biological variability is removed), and an easily reproducible technique to extract the airborne semiochemicals reliably and efficiently.

In recent years, solid-phase microextraction (SPME) has risen in prominence, and this is now considered to be one of the primary techniques for volatile headspace chemical analysis [14]. The SPME technique utilizes a thin fiber coated with a specific extraction phase which can be optimized to selectively extract analytes depending on their properties. Upon exposure of the fiber to the headspace of a sample, the coating phase of the fiber adsorbs airborne compounds selectively, and these compounds then are desorbed in a GC/MS injection port at high temperature for GC/MS analysis. This specific technique of extraction and analysis of volatile compounds is known as headspace-SPME-GC/MS (HSSPME-GC/MS) technology, and it is quicker, more efficient, more sensitive, and less prone to matrix effects when compared to other sampling techniques. Additionally, it has already demonstrated its usefulness in extracting and analyzing the headspace environment of other biological samples, such as marine biota [15], marine aquaria [16], mushrooms [17], and even freeze-thawed insect samples [18]. To our knowledge, no headspace SPME analysis has ever been performed on arachnids, especially for undisturbed living animal samples, and this is potentially a key technique to examine in order to identify airborne semiochemicals.

In this study, we have developed an ultra-sensitive HS-SPME-GC/MS method to extract the airborne biochemicals from the headspace of a spider chamber holding a single living spider. HS-SPME-GC/MS in scan mode was used for the semiochemical identification and for quantifying the compounds. A total of 11 organic compounds present in the headspace of the container holding a single living Loxosceles reclusa spider were identified and quantified.

Loxosceles reclusa (brown recluse spiders, BRS) $[19,20]$ are indigenous American spiders known for bites that can cause painful, slowly healing necrotic ulcers in humans [21-23]. On occasion, these bites may cause severe systemic effects and fatalities [24, 25]. Therefore, identification of the semiochemicals used by these spiders has the potential to significantly improve the health outcomes of many individuals through enabling development of better traps and means of population control within infested houses. Additionally, to demonstrate the usefulness of this method to study other spider species, we confirmed the applicability of this method to another species, Plectreurys tristis. 


\section{Materials and methods}

\section{Chemicals and standard preparation}

Citral (3,7-dimethyl-2,6-octadienal, $98 \%$, is a mixture of the isomers of 1:1 geranial (GER):neral (NER)), 2-methylbutanoic acid (2MB, 98\%), 2,4-dimethylbenzaldehyde (DMB, 90+\%), hexanal (HEX, 98\%), and butanoic acid (BA, 99\%) were purchased from Sigma-Aldrich (St. Louis, Missouri, USA). 4-Methylquinazoline (4MQ, 95\%) was purchased from BLD Pharmatech Co., Limited (Cincinnati, Ohio, USA). Dimethyl sulfone (DMS, 99\%) was purchased from TCI America (Portland, Oregon, USA). 2-Methylpropanoic acid (2MP, 99\%), 3-methylbutanoic acid (3MB, 99+\%), 2-phenoxyethanol (2PE, 99\%), and internal standard ethyl heptanoate $(\mathrm{EtH}, 98+\%)$ were purchased from Fisher Scientific (Waltham, Massachusetts, USA). Physical and chemical characteristics of all of these compounds, as well as abbreviations used for each, are listed in Table 1. Ultrapure water $(18.2 \mathrm{M} \Omega-\mathrm{cm})$ was obtained from a Milli-Q Advantage A10 and Millipore Elix water purification system (Millipore, Billerica, Massachusetts, USA).

\begin{tabular}{lcccc}
\hline Table 1. Physical and chemical characteristics of identified airborne biomolecules & \\
\hline Compound (abbreviation) & $\begin{array}{c}\text { Molecular } \\
\text { weight }\end{array}$ & $\begin{array}{c}\text { Water solubility } \\
(\mathrm{mg} / \mathrm{L})\end{array}$ & $\begin{array}{c}\text { Boiling point } \\
\left({ }^{\circ} \mathrm{C}\right)\end{array}$ & $\begin{array}{c}\text { Vapor pressure } \\
(\mathrm{mmHg})\end{array}$ \\
\hline 2-Methylpropanoic acid (2MP) & 88.11 & 197,000 & 155 & 1.5 \\
Butanoic acid (BA) & 88.11 & 60,000 & 163.7 & 0.43 \\
Hexanal (HEX) & 100.16 & 4,490 & 129 & 10.5 \\
3-Methylbutanoic acid (3MB) & 102.13 & 40,700 & 176 & 0.38 \\
2-Methylbutanoic acid (2MB) & 102.13 & 57,200 & 176 & 0.554 \\
Dimethyl sulfone (DMS) & 94.13 & 55,800 & 238 & 0.1 \\
2,4-Dimethylbenzaldehyde (DMB) & 134.17 & 356 & 220 & $0.110-0.135$ \\
2-Phenoxyethanol (2PE) & 138.16 & 26,000 & 247 & 0.01 \\
Neral (NER) & 152.24 & 59 & 225 & 0.22 \\
Geranial (GER) & 152.24 & 59 & 225 & 0.22 \\
4-Methylquinazoline (4MQ) & 144.17 & $>30 \%$ & 261 & $0-0.5$ \\
Ethyl heptanoate (EtH) & 158.24 & 300 & 188 & 0.68 \\
\hline
\end{tabular}

* Indicates internal standard which is not present in any spider samples.

To prepare standard solutions, the chemicals were mixed and diluted separately to account for different method sensitivities and solubility of the compounds in water. The stock standard solutions were prepared in ultrapure water at the concentrations of $100 \mathrm{mg} / \mathrm{mL}$ of DMS; $20 \mathrm{mg} / \mathrm{mL}$ of $2 \mathrm{MB}, 4 \mathrm{MQ}, 2 \mathrm{MP}, 3 \mathrm{MB}, 2 \mathrm{PE}$, and BA; $2 \mathrm{mg} / \mathrm{mL}$ of HEX; $0.1 \mathrm{mg} / \mathrm{mL}$ of DMB; and $0.05 \mathrm{mg} / \mathrm{mL}$ of NER and GER. Once completely dissolved, we mixed the 2MB, $4 \mathrm{MQ}, 2 \mathrm{MP}, 3 \mathrm{MB}, 2 \mathrm{PE}$, and BA solutions together to create a mixed secondary stock solution of $200 \mathrm{mg} / \mathrm{L}$ each of these components. The stock solutions for the other compounds were used as prepared. Separate stock solutions were used to address the different solubilities of each compound and the greatly varied concentrations of the compounds in the headspace of spiders (specifically in the case of DMS and citral). From here, we employed 
serial dilution to create calibration curves. The internal standard solution was prepared at a concentration of $2 \mathrm{mg} / \mathrm{mL}$ of $\mathrm{EtH}$ in ultrapure water.

\section{Spider collection, housing, and identification}

We collected juvenile and adult Loxosceles reclusa from houses, barns, and outbuildings in south-central Missouri, USA, throughout the duration of the study. We kept the spiders in clear $80-\mathrm{mL}$ polypropylene containers with small holes drilled around the edges of a polystyrene lid. They were housed in spider labs with ambient indoor humidity, with male, female, and juvenile spiders kept in three separate rooms. The temperature in these rooms fluctuated to best replicate the normal thermal variations in the natural BRS indoor household environment. For adult BRS, males and females were distinguished based on the presence of clubbed vs smooth pedipalps. In juveniles, this difference was too small to distinguish. The spiders were fed a diet of infant Acheta domestica crickets once a week throughout the study. Plectreurys tristis spiders were collected in Arizona and then transported to Missouri for analysis. These spiders were kept in similar conditions in a separate room.

\section{HS-SPME sampling}

We sampled the headspace of each live spider in the original container housing the spider in the spider lab using a 23-gauge 100- $\mu$ mpolydimethylsiloxane (PDMS) SPME fiber (Supelco, Bellefonte, Pennsylvania, USA) (Fig. S1). This sampling practice minimizes the potential of induced stress for the spider. The fiber was preconditioned by following the manufacturer's instructions. Spiders were left in their containers for at least a few weeks and allowed to lay web and feed in their containers prior to sampling so that their headspace environment could mimic natural levels and reach equilibrium before testing. Quality control samples were tested the same way as spider samples but with empty spider containers and spider web/food in the containers without spider. It was confirmed that the container alone, BRS web alone, and BRS food alone did not contribute significantly to or interfere with any of the airborne biochemicals spider emitted. Before the first test of each experiment, the fiber was re-conditioned at $240^{\circ} \mathrm{C}$ for $5 \mathrm{~min}$ to clean the fiber before use for sampling. To sample the spider headspace, we inserted the SPME fiber through one of the predrilled air holes on the cap of a spider-holding container and extracted it for $25 \mathrm{~min}$ at a depth of $3 \mathrm{~cm}$ from the top of the container at room temperature. After extraction, the fiber was removed and injected into the GC/MS injection port for a 5-min desorption at $240^{\circ} \mathrm{C}$ inlet temperature. For standard sampling, we washed and thoroughly cleaned the containers with deionized water before adding the standard and sampling after 5 min equilibration, using the same procedure as the spider sampling.

\section{GC/MS method}

We performed GC/MS analysis on an Agilent Technologies Network 6890N GC system coupled with a 5973 Mass Selective Detector with the NIST Mass Spectral Library. Chemstation software (Windows XP) was used for data acquisition and processing. The sample injection port was equipped with a Merlin Microseal High Pressure Septum (Merlin Instrument Company, Half Moon Bay, California) manual SPME device. The GC was equipped with a 30-m HP-5MS capillary column $(0.25 \mathrm{~mm}$ i.d. $\times 0.25 \mu \mathrm{m}$ film thickness). 
The carrier gas was helium at a flow rate of $1.0 \mathrm{~mL} / \mathrm{min}$. The injector temperature was set to $240^{\circ} \mathrm{C}$, and the SPME sample was injected in splitless mode. The oven temperature was programmed to start at $40^{\circ} \mathrm{C}$, where it was held for $2 \mathrm{~min}$. We then increased the temperature up to $150^{\circ} \mathrm{C}$ at $10^{\circ} \mathrm{C} / \mathrm{min}$, followed by an immediate increase to $250^{\circ} \mathrm{C}$ at $20^{\circ} \mathrm{C} / \mathrm{min}$. Transfer line and the MS source temperatures were $260^{\circ} \mathrm{C}$ and $230^{\circ} \mathrm{C}$, respectively. The electron ionization voltage of the mass spectrometer was $70 \mathrm{eV}$. The MSD acquisition was carried out in scan mode at a mass range of 40 to $240 \mathrm{amu}$.

An internal standard calibration method was used for quantification of the airborne biomolecules in order to limit the potential for environmental variation between samples not due to the sample itself. Specifically, the need for air holes in all containers introduced a significant source of variation due to the airborne nature of the analysis and the lack of uniformity in the air holes for each sample. The internal standard was therefore used to normalize for the different rates of effusion through the air holes and other conditions between different samples.

To make standard calibration curves, for each calibration concentration $100 \mu \mathrm{L}$ of standard solution was placed in a clean empty container that matched the spider containers. We chose $100 \mu \mathrm{L}$ arbitrarily due to the similarity in volume between a $100 \mu \mathrm{L}$ drop and a BRS body. The internal standard ( $100 \mu \mathrm{L}$ of $0.02 \mathrm{mg} / \mathrm{mL} \mathrm{EtH})$ was added to the container, together with the standard (separate spot from the standard). After equilibration for $5 \mathrm{~min}$, we performed HS-SPME-GC/MS analysis by the same procedure used for spider headspace sample analysis. The standard peak areas were then compared to this internal standard peak area for each standard to create a calibration curve of analyte/internal standard peak area ratios vs standard concentrations. For BRS samples, $100 \mu \mathrm{L}$ of $0.02 \mathrm{mg} / \mathrm{mL}$ EtH internal standard was placed in the container with a live spider (separate spot from the spider) and analyzed using the HS-SPME-GC/MS method. This same internal standard was used for all the spider samples, blanks, and calibration standards.

\section{Identification of airborne biochemicals emitted from BRS}

Compounds from BRS were first tentatively identified by comparing the mass spectra of peaks uniquely found in the headspace sample of BRS with compounds in the mass spectral library. We then used standards to confirm our identification of these chemicals by placing the chemical standard in a clean empty spider container and analyzing the standard using the same optimized HS-SPME-GC/MS sampling and analysis method. Retention times and mass spectral data were compared between the spider samples and the standards to confirm identity.

\section{Quality control and statistical evaluation}

Good quality control procedures were included during the method development and spider sample analyses. We analyzed blanks (empty clean spider container) each time before analyzing any standard or sample to check contamination and after analysis to check carryover. An internal standard was used to minimize the impacts from any variation of sampling and environment. Calibration curves were conducted (at least 6 points) or checked (using two different concentrations of standard mixture) each time before analyzing any spider sample to ensure good calibration linearity and sensitivity. All the calibration 
standards and spider samples were analyzed at least in duplicate to ensure good precision. We also tested spike recovery by spiking a known amount of standard in the container with a live spider to ensure the accuracy of the method. Finally, in order to determine the wider applicability of this method to other spider species, we collected and analyzed the headspace samples of another spider species, P. tristis, using the developed method to compare differences between the genders and species. We used Student's $t$ test to evaluate the difference between different categories of spiders. A $P$ value of 0.05 or smaller indicated a significant difference between groups.

\section{Results}

\section{Method optimization}

We initially tested mass-to-charge ratios ranging from 25 to 400 amu in a wide mass range to identify all possible relevant spider airborne biochemicals. However, we found out all the airborne biomolecules under the experimental conditions were in a mass range of $<240$ amu. Therefore, a mass scan range of 40 to 240 amu was selected for mass detection.

Preliminary experimentation with different SPME fibers for spider headspace samples led us to choose a 100- $\mu \mathrm{m}$ PDMS fiber because of its ability to extract the widest range of volatile compounds from spiders (Fig. S2) at reproducible levels. A total of 17 compounds were found in the headspace of BRS. Three different types of SPME fibers, $100 \mu \mathrm{m}$ PDMS, $75 \mu \mathrm{mCarboxen} / \mathrm{PDMS}$, and $60 \mu \mathrm{m}$ PEG fibers, were evaluated for their performance in detecting each of these compounds. While compounds with low retention times were more detectable using the Carboxen/PDMS fiber and a select few compounds were also more efficiently extracted using the PEG fiber, the PDMS fiber showed the best performance because of its efficient extraction for all the spider analytes identified. Therefore, PDMS fiber was selected for all subsequent experiments.

The other conditions, including SPME extraction time (tested from 15 to $45 \mathrm{~min}$ ), the depth of fiber exposure into the spider container, desorption temperature and time, GC separation columns and conditions, including temperature gradient and carrier gas helium flow rate, and mass detection conditions, were all tested and optimized. Although a DBFFAP (nitroterephthalic acid-modified polyethylene glycol) capillary GC column (30 m × $0.25 \mathrm{~mm}, 0.25 \mu \mathrm{m}$ film) gets good resolution and good peak for some more polar compounds, the HP-5MS column resolved and detected more compounds from headspace of spiders; therefore, a HP-5MS column was selected for all the subsequent experiments. The optimized conditions were used in the final method as stated in the "Materials and methods" section.

\section{Airborne biochemicals identified from BRS}

A total of 17 compounds were found in the headspace samples of BRS as shown in Figure 1a. We were able to tentatively identify 11 of these 17 compounds by matching with the mass spectra of the NIST library. The 6 unidentified compounds tended to have longer retention times and mass spectra that did not readily match any known compound in the NIST library. 


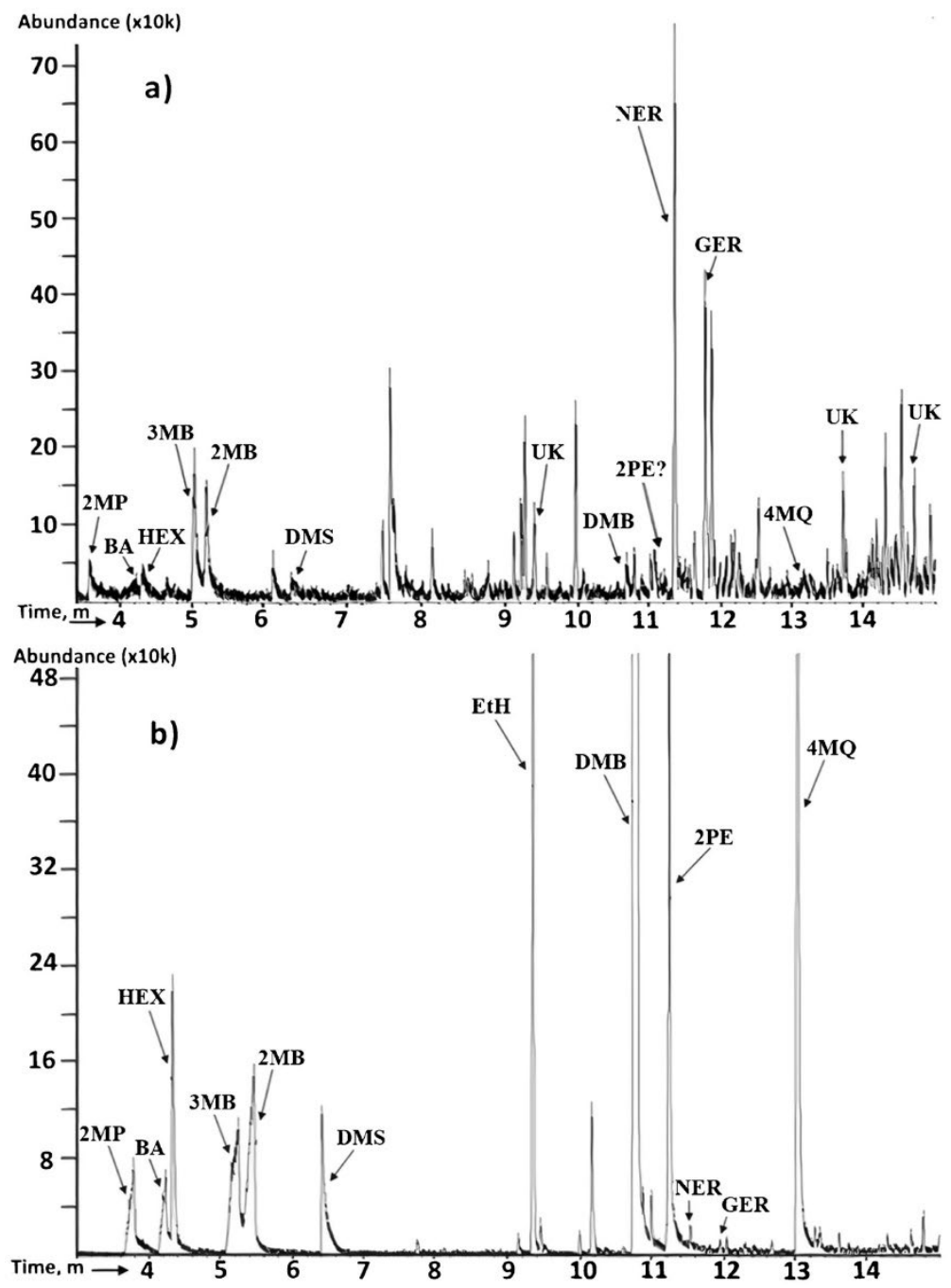

Figure 1. Representative chromatograms of (a) airborne biochemicals in the headspace sample of a BRS and (b) a standard mixture. All unlabeled peaks were also present in samplings of only the empty containers and so were assumed to be of nonbiological origin.

In order to further confirm the identity of the biochemicals from BRS, we purchased all the 11 chemicals. These standards were prepared and then analyzed using the same HSSPME-GC/MS method. A representative chromatogram of a standard mixture is shown in Figure $1 \mathrm{~b}$. The peak identities were confirmed by matching both the retention time and mass spectrum to the spider-produced compound peaks. Each of the 11 peaks was confirmed to match the standard obtained through retention time, mass spectrum from the standard, and mass spectrum of the NIST library. Figure 2a shows an example of the neral compound mass spectrum in a BRS sample, and the mass spectrum of the neral standard for comparison is shown in Figure 2b. All of the 11 airborne biochemicals were identified using this process. 

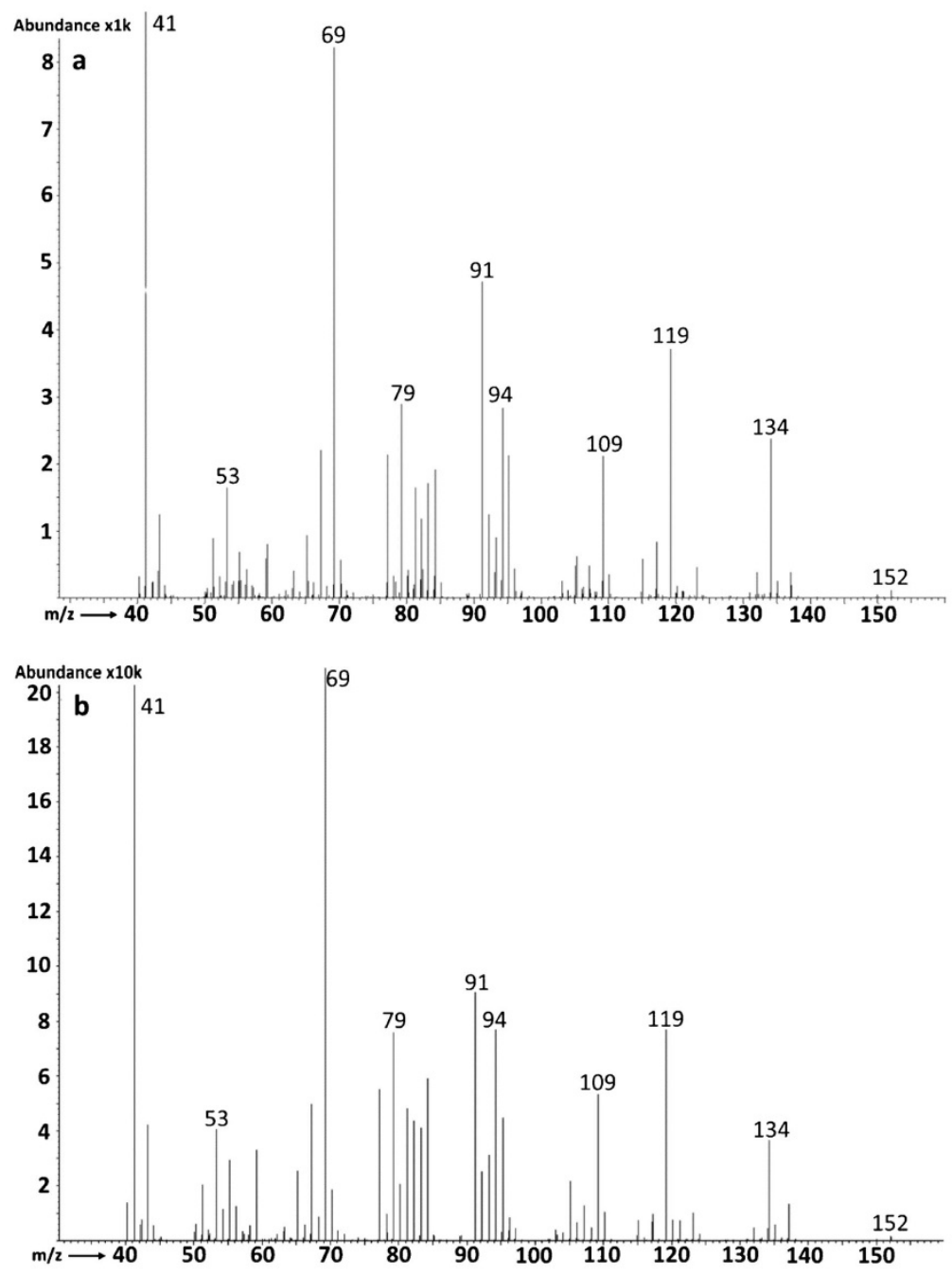

Figure 2. Representative mass spectra from the neral (retention time, $11.55 \mathrm{~min}$ ) peaks of (a) a BRS sample and (b) a standard mixture.

\section{Method performance}

After optimization of all method parameters, we then checked method performance. Because of the large sensitivity range of different biochemicals from BRS samples, different concentration ranges of the biochemicals were used for making the calibration curves. A minimum of 5 points ( 5 concentrations) were collected for each compound in its calibration curve, and the average signals were used to construct the calibration curve from at least triplicate injections at each concentration. Initial testing without using an internal standard found strong linearity in curves run on the same day, but when run on different days, the peak area varied for the same standard concentration. When an internal standard was added to each sample, and the standard curves were constructed based on the analyte-tointernal standard ratio vs concentration, good linearity was observed both between days 
and on the same day. The calibration linearity and the corresponding concentration range for each biochemical are tabulated in Table 2. All the calibration curves of the biochemicals show very good linearity $\left(R^{2}>0.93\right)$. These concentration ranges were chosen to include the peak area from all spider samples tested. All were still linear at the maximum concentration tested, and it is possible that the linear range extends higher than the listed concentration.

\begin{tabular}{lccccc}
\hline \multicolumn{2}{l}{ Table 2. Method performance characteristics for analytes } & & & \\
& $\begin{array}{c}\text { Retention } \\
\text { time }(\mathrm{min})\end{array}$ & $\begin{array}{c}\text { Linear } \\
\text { range } \\
(\mathrm{mg} / \mathrm{L})\end{array}$ & $R^{2}$ & $\begin{array}{c}\text { Limit of } \\
\text { detection } \\
(\mathrm{mg} / \mathrm{L})^{*}\end{array}$ & $\begin{array}{c}\text { Spike } \\
\text { recovery } \\
(\%)\end{array}$ \\
Compound (abbreviation) & 3.7 & $10-200$ & 0.9758 & 10 & 77.01 \\
2-Methylpropanoic acid (2MP) & 4.15 & $25-200$ & 0.9765 & 10 & 138.7 \\
Butanoic acid (BA) & 4.3 & $5-200$ & 0.9549 & $<0.05$ & 117.8 \\
Hexanal (HEX) & 5.1 & $10-200$ & 0.9870 & 10 & 114.8 \\
3-Methylbutanoic acid (3MB) & 5.3 & $10-200$ & 0.9815 & 10 & 92.28 \\
2-Methylbutanoic acid (2MB) & 6.5 & $500-10,000$ & 0.9777 & 200 & 91.31 \\
Dimethyl sulfone (DMS) & 10.9 & $0.05-200$ & 0.9999 & 0.01 & 95.31 \\
2,4-Dimethylbenzaldehyde (DMB) & 11.3 & $10-200$ & 0.9337 & 0.1 & 72.09 \\
2-Phenoxyethanol (2PE) & 11.55 & $0.005-5$ & 0.9973 & $<0.005$ & 106.6 \\
Neral (NER) & 11.95 & $0.005-5$ & 0.9892 & $<0.005$ & 99.64 \\
Geranial (GER) & 13.05 & $0.5-200$ & 0.9533 & $<0.5$ & 77.07 \\
4-Methylquinazoline (4MQ) & & & & &
\end{tabular}

* Equivalent concentration in calibration liquid.

The limit of detection (LOD) was defined as a peak with a signal-to-noise ratio $(\mathrm{S} / \mathrm{N})$ of about 3 for each compound and is shown for each compound in Table 2. These data represent the concentrations in the liquid placed in the spider containers, not headspace gas concentrations. Therefore, the quantitation method here is a relative quantification, as the SPME sampling in general, not absolute concentration quantification in the headspace gas phase.

We also performed spike recovery experiments by spiking $100 \mu \mathrm{L}$ aliquot of standards into the container with a live spider. Due to the large difference in levels of the airborne chemicals detected in BRS samples and fairly wide range of sensitivities for different analytes of the method, spike recovery experiments were performed with different concentrations of analytes, close to the levels in spider headspace samples. The results are shown in Table 2. The spike recoveries for all the biochemicals are in the range of $72 \%$ to $118 \%$, except BA with a spike recovery $138.65 \%$.

\section{Comparison of airborne biochemicals in Loxosceles reclusa and Plectreurys tristis}

After identifying 11 of the spider-produced chemicals present in the headspace of BRS and optimizing our method's performance, we then applied this method to quantify these airborne biochemical concentrations in the headspace of male and female BRSs. To determine the broader applicability of this method to other spider species, we also analyzed these airborne biomolecules in the headspace of $P$. tristis spiders using the same procedure as 
BRS samples. The difference in concentration of these biochemicals between male and female BRSs as well as between recluse and P. tristis spiders was evaluated using Student's $t$ test to compare between the male and female BRSs and different spider species.

A total of 4 male BRS, 4 female BRS, and 4 P. tristis spiders were tested after spiking with the internal standard. The average headspace concentrations are listed in Table 3 for these different spider types, and also included is a $P$ value to determine whether the difference between male and female BRS and between Loxosceles and Plectreurys is statistically significant.

Table 3. Comparison of airborne biochemical concentrations among different spider species and genders

\begin{tabular}{|c|c|c|c|c|c|}
\hline \multirow[b]{2}{*}{ Compound } & \multicolumn{3}{|c|}{ Average concentration $(\mathrm{mg} / \mathrm{L})^{*}$} & \multicolumn{2}{|c|}{ Statistical significance $(P)$} \\
\hline & $\begin{array}{l}\text { Male BRS } \\
\quad(n=4)\end{array}$ & $\begin{array}{c}\text { Female BRS } \\
\quad(n=4)\end{array}$ & $\begin{array}{l}\text { Plectreurys } \\
\quad(n=4)\end{array}$ & $\begin{array}{c}\text { Male } B R S \text { vs. } \\
\text { female } B R S\end{array}$ & BRS vs Plec. \\
\hline $2 \mathrm{MP}$ & $12.49(22.6 \%)$ & $14.03(27.7 \%)$ & $27.00(9.7 \%)$ & 0.566 & $<0.001^{* *}$ \\
\hline BA & $32.15(4.6 \%)$ & $30.03(9.2 \%)$ & $35.81(14.5 \%)$ & 0.225 & $0.049^{* *}$ \\
\hline HEX & $12.09(2.4 \%)$ & $11.73(8.5 \%)$ & $13.42(10.9 \%)$ & 0.515 & $0.033^{* *}$ \\
\hline $3 \mathrm{MB}$ & $62.15(38.3 \%)$ & $51.42(60.0 \%)$ & $237.0(94.5 \% \wedge)$ & 0.602 & $0.040^{* *}$ \\
\hline $2 \mathrm{MB}$ & $27.92(26.0 \%)$ & $21.90(54.4 \%)$ & $75.09(69.3 \% \wedge)$ & 0.421 & $<0.001^{* *}$ \\
\hline DMS & $1,773(21.2 \%)$ & $1,062(29.6 \%)$ & $2,247(27.7 \%)$ & $0.027^{* *}$ & $0.030^{* *}$ \\
\hline DMB & $<$ LOD & $<$ LOD & $<$ LOD & - & - \\
\hline $2 \mathrm{PE}$ & $<$ LOD & $<$ LOD & $<\mathrm{LOD}$ & - & - \\
\hline NER & $0.063(18.3 \%)$ & $0.061(9.9 \%)$ & $0.069(8.8 \%)$ & 0.673 & 0.208 \\
\hline GER & $0.113(9.1 \%)$ & $0.100(3.7 \%)$ & $0.115(8.3 \%)$ & $0.062^{* * *}$ & 0.183 \\
\hline $4 \mathrm{MQ}$ & $9.37(11.0 \%)$ & $8.98(47.8 \%)$ & $9.20(20.2 \%)$ & 0.866 & 0.977 \\
\hline
\end{tabular}

Numbers in parentheses indicate relative standard deviation.

$<\mathrm{LOD}=$ below limit of detection.

* Equivalent concentration in calibration liquid.

** Indicates a statistically significant difference between two spider categories $(P<0.05)$.

*** Indicates approaching statistical significance $(P<0.1)$.

$\wedge$ The higher variations are due to the substantial difference between adults $(n=2)$ and juveniles $(n=2)$ of Plectreurys spiders in addition to the fact that both $3 \mathrm{MB}$ and $2 \mathrm{MB}$ exhibit relatively high variation between spider samples in general.

Finally, in order to compare our quantitation and comparison method to a currently established method of comparing the chemical content of spider samples, we also compared the relative abundance of each of the 17 spider-produced airborne compounds (including the 6 unidentified compounds) to see if there was a statistically significant different in relative abundance. This method of quantification is the same type used by Xiao et al. (2009) [9], and it involves taking the peak area of a single spider-produced analyte and dividing it by the peak area of all produced analytes to get a percentage value. The comparisons and $P$ values between the same groups as above are shown in Table 4, with the unknown compounds listed by their retention times. This type of quantitation has the benefit of being simpler because of the ability to avoid directly quantifying the compounds, and in spiders of the same species, direct comparisons can easily be made to assess if one 
group of spiders is producing a relatively larger amount of a compound than another. Additionally, this allows for a comparison of unidentified compounds whose concentrations are otherwise unquantifiable. However, the lack of ability to directly calculate concentrations using this method is a downside, and in a comparison between two samples with largely different chemical headspace environments (which might be the case when comparing two different spider species), there could be a relative abundance difference due to the extreme change in quantity of other analytes and not specifically a direct change in actual analyte concentration.

Table 4. Comparison of analyte concentration in the headspace of male BRS, female BRS, and Plectreurys spiders using the relative abundance method [9]

\begin{tabular}{|c|c|c|c|c|c|}
\hline \multirow[b]{2}{*}{ Compound } & \multicolumn{3}{|c|}{ Relative abundance (\%) } & \multicolumn{2}{|c|}{ Statistical significance $(P)$} \\
\hline & $\begin{array}{l}\text { Male } B R S \\
\quad(n=4)\end{array}$ & $\begin{array}{c}\text { Female } B R S \\
\quad(n=4)\end{array}$ & $\begin{array}{l}\text { Plectreurys } \\
\quad(n=4)\end{array}$ & $\begin{array}{l}\text { Male Lox. vs. } \\
\text { female Lox. }\end{array}$ & Lox. vs Plec. \\
\hline $2 \mathrm{MP}$ & $5.86(28.1 \%)$ & $6.76(25.2 \%)$ & $7.00(22.1 \%)$ & 0.477 & 0.499 \\
\hline $\mathrm{BA}$ & $4.50(22.5 \%)$ & $2.67(82.4 \%)$ & $3.58(10.3 \%)$ & 0.181 & 0.992 \\
\hline HEX & $5.70(14.2 \%)$ & $4.60(80.1 \%)$ & $5.20(15.4 \%)$ & 0.581 & 0.971 \\
\hline $3 \mathrm{MB}$ & $25.58(20.4 \%)$ & $30.79(8.3 \%)$ & $35.81(21.9 \%)$ & 0.123 & $0.059^{* *}$ \\
\hline $2 \mathrm{MB}$ & $19.73(10.7 \%)$ & $21.57(7.1 \%)$ & $23.12(1.7 \%)$ & 0.209 & 0.036 \\
\hline DMS & $9.64(40.2 \%)$ & $3.52(71.1 \%)$ & $6.62(29.1 \%)$ & $0.038^{*}$ & 0.987 \\
\hline RT 9.65 & $5.66(11.5 \%)$ & $5.39(66.1 \%)$ & $4.13(15.8 \%)$ & 0.886 & 0.288 \\
\hline DMB & $<$ LOD & $<$ LOD & $<\mathrm{LOD}$ & - & - \\
\hline $2 \mathrm{PE}$ & $<$ LOD & $<$ LOD & $<\mathrm{LOD}$ & - & - \\
\hline NER & $1.95(77.0 \%)$ & $2.06(18.3 \%)$ & $1.21(29.4 \%)$ & 0.892 & 0.167 \\
\hline RT 11.6 & $4.12(4.8 \%)$ & $2.89(30.7 \%)$ & $2.72(70.2 \%)$ & $0.036^{*}$ & 0.345 \\
\hline GER & $2.08(48.0 \%)$ & $1.61(42.0 \%)$ & $1.14(38.3 \%)$ & 0.467 & 0.151 \\
\hline RT 12.4 & $2.63(42.8 \%)$ & $1.93(14.0 \%)$ & $1.46(41.6 \%)$ & 0.274 & 0.116 \\
\hline $4 \mathrm{MQ}$ & $2.78(17.9 \%)$ & $3.64(20.4 \%)$ & $1.49(48.9 \%)$ & 0.146 & $0.004^{*}$ \\
\hline RT 13.82 & $1.91(44.8 \%)$ & $3.55(73.7 \%)$ & $0.60(86.5 \%)$ & 0.279 & $0.068^{* *}$ \\
\hline RT 13.9 & $2.15(34.2 \%)$ & $3.15(68.3 \%)$ & $1.28(27.0 \%)$ & 0.413 & 0.125 \\
\hline RT 14.4 & $6.41(41.8 \%)$ & $7.83(44.4 \%)$ & $4.65(40.8 \%)$ & 0.566 & 0.176 \\
\hline
\end{tabular}

Numbers in parentheses indicate relative standard deviation. Unidentified compounds are still produced by spiders at detectable levels and so must be included in this \% relative abundance study. Unidentified compounds are listed by their retention times (RT 9.65 means the unidentified peak that elutes at $9.65 \mathrm{~min}$, for example).

$<$ LOD = below limit of detection.

${ }^{*}$ Indicates a statistically significant difference between two spider categories $(P<0.05)$.

** Indicates approaching statistical significance $(P<0.1)$.

\section{Discussion}

In this paper, we described a novel technique to examine the airborne biochemicals of spiders and potentially other small creatures using HS-SPME-GC/MS. We demonstrated the ability of this method to target, identify, and quantitate specific analytes in one species, Loxosceles reclusa, and we then showed the potential for wider application of this method 
by using it to detect the same compounds in Plectreurys tristis spiders. We developed a method to quantify the concentration of the analytes in the headspace of these spiders relative to a liquid-phase standard curve. This provides more specific information that can be used to analyze differences between different spiders and different spider categories as well as different species.

While our method is able to determine the concentrations of nearly a dozen different analytes in the headspace of a single live spider in a nonenclosed $80-\mathrm{mL}$ container at equilibrium, there could be issues when attempting to directly apply these concentrations to field studies in nature. There is a concern that these environments are artificial habitats that differ from the standard spider habit, which might directly impact the spiders' semiochemical production behavior. The enclosed space of the container matches the enclosed habitats that these spiders often find while within structures, but there is a concern that too much movement of the container or exposure to light might influence the results. Prior to and during our experiments, we minimized these environmental impacts by keeping the spiders in a dark, static environment, except the time of headspace sampling when it needs to be on the lab bench. The relative volume of headspace to spider suggests that it would take at least a few hours for changes in semiochemical production by the spider to manifest in a change in the headspace equilibrium, so any stress caused by movement during internal standard addition and headspace extraction should not significantly impact the headspace environment during the 25 min of extraction.

Additionally, the fact that it took a period of several days for the headspace concentration to reach equilibrium means that these spiders likely produce much lower concentrations of each of these chemicals in a given period of time, and additional studies will be needed to determine the rate of chemical production and release from the bodies of the spiders directly. However, since the containers are a nonenclosed environment with air holes in the top cover, it is reasonable to suggest that the final internal headspace concentration of each compound will be proportional to the rate of that compound's release from the spider body, and it will represent the point at which the release from the spider's body is equivalent to the rate of effusion through the air holes. Therefore, the calculated concentrations using this method are still proportional to the analyte release rate from the spider and can thus be used to confidently compare relative rates between species. The use of the internal standard as a normalization factor allows researchers to control for possible different rates of effusion due to experimental sample variations (such as different amounts or sizes of air holes between different samples) since sample variation will have an impact on the concentration of internal standard present as well.

Some of the identified chemicals in the spider headspace are also used for signaling by other species. Citral (including both isomers, neral and geranial) is a primary component of the Nasonov pheromone used for location signaling in honeybees. Additionally, 4-methylquinazoline is a mating pheromone in some parasitic wasp species, and 2-methylpropanoic acid, 2-methylbutanoic acid, and dimethyl sulfone have all been identified as signaling compounds in some spider silk samples. Additional study of other species that produce these compounds using this SPME-GC/MS method might be helpful to compare their headspace environments to BRS and $P$. tristis spiders, and the known concentrations 
being produced by these species might enable a better understanding of the innate chemical concentrations being produced by these spiders and others.

This study also revealed some interesting trends of analyte production among the BRS population that warrant further exploration in additional study. One particular compound of note was 2-phenoxyethanol. Although this compound was present with a very small to nondetectable level in the majority of BRS samples, it was noticed in preliminary experiments that BRS whose headspace was tested within a few weeks of mating with another BRS expressed this compound at greatly increased concentrations. Additionally, 4-methylquinazoline was seemingly only significantly present among virgin BRS, and both isomers of citral (neral and geranial) were produced at much higher levels during the BRS mating season (approximately June through September). All spider samples included in our spider analysis were adult spiders who were not recently exposed to other spiders and who remained in their containers at room temperature conditions for at least 1 month before testing so that variables arising from these factors could be controlled and the specific gender-dependent (in the case of BRS) or species-dependent (between Loxosceles reclusa and Plectreurys tristis) differences in headspace environment could be isolated and demonstrated. Additionally, all spiders included in the comparison analysis were sampled outside of the mating season. Since these conditions and prior treatments were not conducive to the production of significant concentrations of some compounds for the reasons explained above, there are potentially larger and more significant trends in their production which are not included in our exploratory analysis. There are many other factors that this method can be used to examine, and we are currently studying how season, gender, maturity, and mating status impact these airborne biochemical expressions of BRS using this sampling method.

It should also be indicated that this method used scan-mode mass spectrometry to both identify and quantify the 11 compounds rather than using selective ion monitoring (SIM) mode to quantify. Indeed, while scan mode does work as a method of quantification, the method sensitivity and accuracy could be improved through SIM mode for quantification for each identified chemical. We plan to conduct further experiments to optimize the quantification using SIM mode signaling to monitor the identified biochemicals and analyze more insect species.

Finally, an analysis of the data in Tables 3 and 4, together with the chromatograms of Loxosceles reclusa and Plectreurys tristis (Fig. S3), reveals a couple of interesting results. We can see that there is a clear difference in airborne biochemicals between the two species, and this difference is especially apparent when comparing relative concentrations with our standard curve rather than relative peak areas. Since we would expect volatile signaling patterns to differ between species, this demonstrates a clear advantage for our standard curve calibration method over the relative abundance method in identifying distinctions between the groups, and the additional information regarding exact liquid-phase concentrations which produce a headspace signal relative to a spider at equilibrium could be helpful in designing future behavioral tests.

When comparing within the same species, though, the two methods have comparable effectiveness. Both the standard comparison method and relative abundance method serve to identify significant difference of headspace chemical concentration between male and 
female BRS. The results indicated that dimethyl sulfone concentration showed prominent gender difference. Additionally, due to the ability of the raw abundance method to compare unknown peaks, differences approaching significance were discovered between the two sexes in the production of unknowns with a retention times of 11.6 and $13.82 \mathrm{~min}$. Identification of these two unknown biochemicals should warrant their usefulness as potential sexual pheromone and/or special functional biomarkers. This reinforces the usefulness of the relative abundance method for intraspecies analysis and comparison, although the additional concentration data would still be helpful in conducting further behavioral assays.

The biggest advantage of this method over currently established procedures is its nondestructive sampling ability for a single live spider, which enables direct observation of a single individual over time and therefore allows for correlation of maturity and mating status with the biochemicals emitted. This is particularly useful for the identification of pheromones and other signaling semiochemicals which can control how populations interact and breed, as chemical trends in individuals can now be much more easily matched to changes in behavior through repeated sampling of the same individual. Additionally, the use of a standard curve in our quantitation method facilitates comparison of chemical concentrations between different spider species. The published relative abundance method assumes that the same compounds will be present at roughly similar concentrations and tends to fail when large changes in the concentration of a single compound are observed between sample categories. A standard curve allows for quantification which is independent of the other chemicals observed in the headspace and removes some complications in interspecies comparison.

While this method optimization process was specific to the two species tested, this optimization procedure can be used to develop similar nondestructive SPME-GC/MS sampling and quantitation methods for other spiders. Additionally, the described quantitation method can be further improved in more specific and focused analyses by using SIM mode with mass spectrometry to quantitate headspace concentrations. We therefore hope that this method can be used as a baseline model to help develop SPME-GC/MS sampling and quantitation experiments for other spider and insect species so that we can much more quickly improve our understanding of the relationships between biochemical signals and behavior in many different species. It may be useful to notice that a limitation is inherent in the technique presented. The method allows distinction of isomers-for example, geranial and neral-however differentiation of enantiomers is not possible without additional techniques. The different chirality of the identified biochemicals may have different biological functions.

\section{Conclusions}

In this study, we developed a novel HP-SPME-GC/MS method for analyzing airborne biochemicals in the headspace of a single live spider. Using this nondestructive sampling method, we have identified and quantified 11 airborne biochemicals emitted by BRS and another spider species. The intraspecies and interspecies biochemical comparisons used both this standard curve-based quantitation method and the previously established 
relative abundance method [9]. Our finalized method was able to identify and quantify biochemicals with a large variety of functionalization and physical properties, suggesting that it might be very useful in the sampling of many other spider species. We observed significant differences between the two spider species in many of the directly monitored compounds using both quantitation methods, and we also observed some significant differences between different sexes of the same species. This demonstrates the usefulness of this method in identifying unique and potentially behavior-altering chemical patterns between species and even identifying separate patterns in the same species, which might be indicative of a compound related to mating or territorial marking.

Acknowledgments - The project was supported by Ozark Biomedical Initiative, a joint effort of Phelps Health Hospital, Missouri University of Science and Technology, and SpiderTek, LLC, Rolla, Missouri. The authors appreciate the assistance of Casey Burton, PhD, Medical Research Director of Phelps Health Hospital.We also appreciate the help of Alex Cristea and Dr. Haiting Zhang for help in developing methods for this project.

Availability of data and material - The data used in this study are available from the corresponding author upon reasonable request.

Conflict of interest - The authors declare no competing interests.

\section{References}

1. Schulz S. Spider pheromones - a structural perspective. J Chem Eco. 2013. https://doi.org/ 10.1007/s10886-012-0231-6.

2. Fischer A. Chemical communication in spiders-a methodological review. J Arachnol. 2019. https://doi.org/10.1636/0161-8202-47.1.1.

3. Kasumovic MM, Andrade MCB. Discrimination of airborne pheromones by mate-searching male western black widow spiders (Latrodectus hesperus): species- and population-specific responses. Can J Zool. 2004. https://doi.org/10.1139/Z04-081.

4. Hoefler CD, Moore JA, Reynolds KT, Rypstra AL. The effect of experience on male courtship and mating behaviors in a cellar spider. Am Midl Nat. 2010. https://doi.org/10.1674/0003-0031163.2.255.

5. Hutton BA, Rypstra AL. Courtship and the use of pheromones by Pholcus manueli (Araneae: Pholcidae). J Arachnol. 2016. https://doi.org/10.1636/J15-38.1.

6. Scott CE, Anderson AG, Andrade MCB. A review of the mechanisms and functional roles of male silk use in spider courtship and mating. J Arachnol. 2018. https://doi.org/10.1636/JoA-S-17093.1.

7. Yew JY, Chung H. Insect pheromones: an overview of function, form, and discovery. Prog Lipid Res. 2015. https://doi.org/10.1016/j.plipres.2015.06.001.

8. Schulz S, Toft S. Identification of a sex pheromone from a spider. Science. 1993. https://doi.org/ 10.1126/science.260.5114.1635.

9. Xiao Y-H, Zhang J-X, Li S-Q. A two-component female-produced pheromone of the spider Pholcus beijingensis. J. Chem. Eco. 2009. https://doi.org/10.1007/s10886-009-9660-2. 
10. Jerhot E, Stoltz JA, Andrade MCB, Schulz S. Acylated serine derivatives: a unique class of arthropod pheromones of the Australian redback spider, Latrodectus hasselti. Angew. Chem. Int. Ed. Engl. 2010. https://doi.org/10.1002/anie.200906312.

11. Prouvost O, Trabalon M, Papke M, Schulz S. Contact sex signals on web and cuticle of Tegenaria atrica (Araneae, Agelenidae). Arch Insect Biochem Physiol. 1999. https://doi.org/10.1002/(SICI) 1520-6327(1999)40:4<194::AID-ARCH4>3.0.CO;2-P.

12. Papke MD, Riechert SE, Schulz S. An airborne female pheromone associated with male attraction and courtship in a desert spider. Anim Behav. 2001. https://doi.org/10.1006/anbe.2000.1675.

13. Chinta SP, Goller S, Lux J, Funke S, Uhl G, Schultz S. The sex pheromone of the wasp spider Argiope bruennichi. Angew Chem Int Ed Engl. 2010. https://doi.org/10.1002/anie.200906311.

14. Kumar A, Gaurav, Malik AK, Tewary DK, Singh B. A review on development of solid phase microextraction fibers by sol-gel methods and their applications. Anal. Chim. Acta. 2008. https://doi .org/10.1016/j.aca.2008.01.028.

15. Noventa S, Barbaro J, Formalewicz M, Gion C, Rampazzo F, Brusà RB, Gabellini M, Berto D. A fast and effective routine method based on HS-SPME-GC-MS/MS for the analysis of organotin compounds in biota samples. Anal Chim Acta. 2015. https://doi.org/10.1016/j.aca.2014.11.028.

16. Shi H, Adams C. Occurrence of formation of trihalomethanes in marine aquaria studied using solid-phase microextraction gas chromatography-mass spectrometry. Water Environ Res. 2012. https://doi.org/10.2175/106143012x13280358613381.

17. Aisala H, Sola J, Hopia A, Linderborg KM, Sandell M. Odor-contributing volatile compounds of wild edible Nordic mushrooms analyzed with HS-SPME-GC-MS and HS-SPME-GC-O/FID. Food Chem. 2019. https://doi.org/10.1016/j.foodchem.2019.01.053.

18. Chen J. Freeze-thaw sample preparation method improves detection of volatile compounds in insects using headspace solid-phase microextraction. Anal Chem. 2017. https://doi.org/10.1021/ acs.analchem.7b01622.

19. Platnick NI. Advances in spider taxonomy, 1988-1991: with synonymies and transfers, 19401980. Arachnol Mitt. 1993. https://doi.org/10.5431/aramit0611.

20. Parks J, Stoecker WV, Kristensen C. Observations on Loxosceles reclusa (Araneae, Sicariidae) feeding on short-horned grasshoppers. The J of Arachnology. 2006. https://doi.org/10.1636/S04-32.1.

21. Atkins JA, Wingo CW, Sodeman WA. Probable cause of necrotic spider bite in the Midwest. Science. 1957. https://doi.org/10.1126/science.126.3263.73.

22. Payne KS, Schilli KD, Meier K, Rader RK, Dyer JA, Mold JW, Green JA, Stoecker WV. Extreme pain from brown recluse spider bites: model for cytokine-driven pain. JAMA Dermatol. 2014. https://doi.org/10.1001/jamadermatol.2014.605.

23. Swanson DL, Vetter RS. Bites of brown recluse spiders and suspected necrotic arachnidism. N Engl J Med. 2005. https://doi.org/10.1056/NEJMra041184.

24. Anderson PC. Spider bites in the United States. Dermatol Clin. 1997. https://doi.org/10.1016/ s0733-8635(05)70438-1.

25. Schilli KD, Rader RK, Payne KS, Green JA, Stoecker WV. Obtundation and myocardial infarction in a case of systemic loxoscelism. Mo Med. 2014;11:143-7. 


\section{Supplementary Information}

\section{Identification and Quantification of 11 Airborne Biochemicals Emitted by the Brown Recluse and Another Primitive Hunting Spider Using Head Space-Solid Phase Microextraction-GC/MS}

Zachary Foulks, ${ }^{1}$ Jennifer Parks, ${ }^{1,2}$ William Stoecker, ${ }^{2}$ Charles Kristensen, ${ }^{3}$ Eileen A. Hebets, ${ }^{4}$ Honglan Shi*, 1

${ }^{1}$ Department of Chemistry, Missouri University of Science and Technology, Rolla, MO 65409, USA

${ }^{2}$ The Dermatology Center and SpiderTek, Rolla, MO 65401, USA

${ }^{3}$ SpiderPharm, Yarnell, AZ 85362, USA

${ }^{4}$ School of Biological Sciences, University of Nebraska, Lincoln, NE 68588, USA

*Corresponding author

Department of Chemistry, Missouri University of Science and Technology 400 W 11th Street, Rolla, MO 65409, USA

Telephone: (573)-341-4420

Fax: (573)-341-4377

E-mail: honglan@mst.edu

ORCID: 0000-0002-0801-5683

\section{Table of Contents}

Figure S1. Spider being sampled with SPME fiber.

Figure S2. Relative Signals of Spider-Produced Volatile Compounds Extracted with Different SPME fibers.

Figure S3. A representative chromatogram of Plectreurys tristis spider with Internal Standard. 


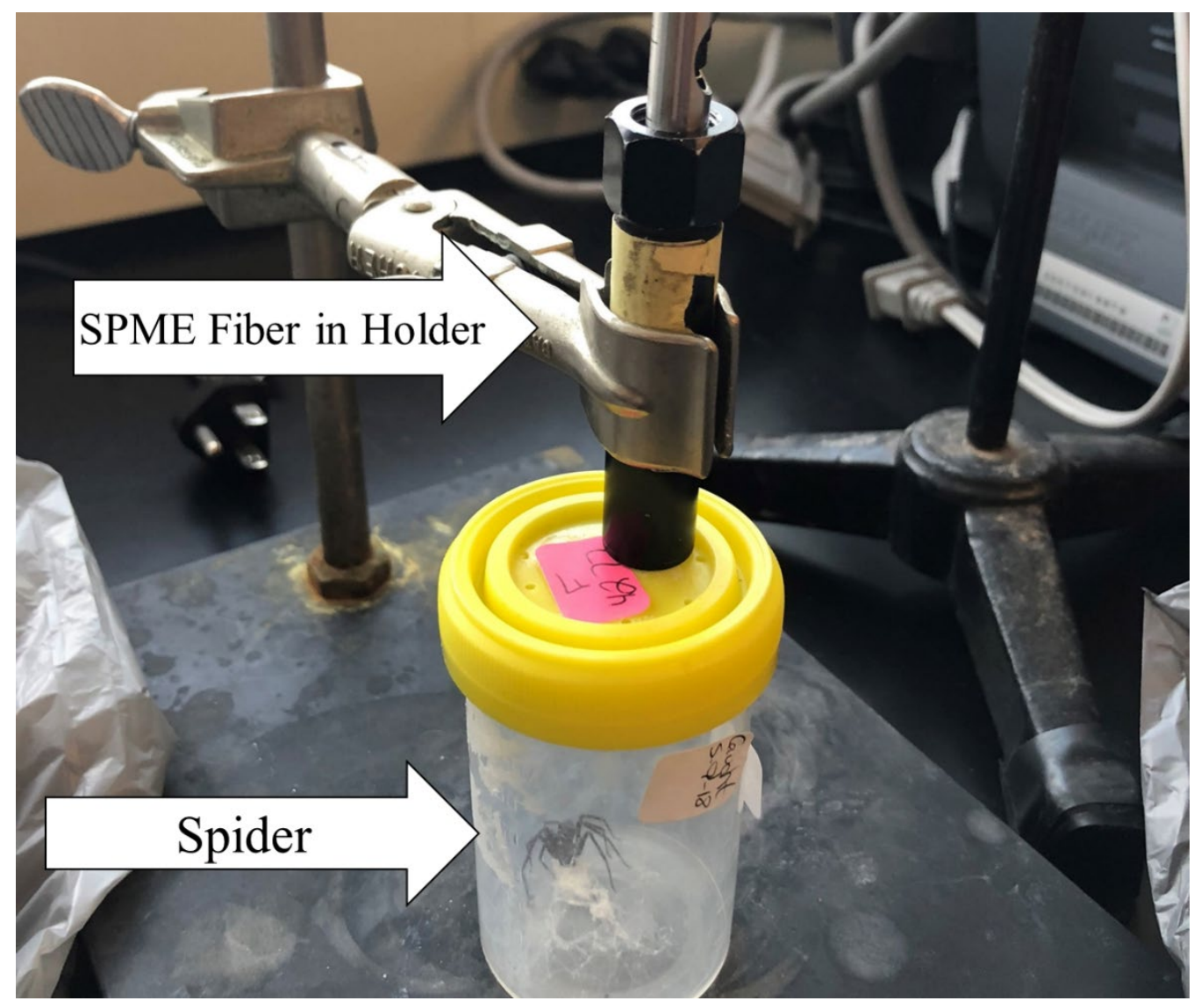

Figure S1. Spider being sampled with SPME fiber. 


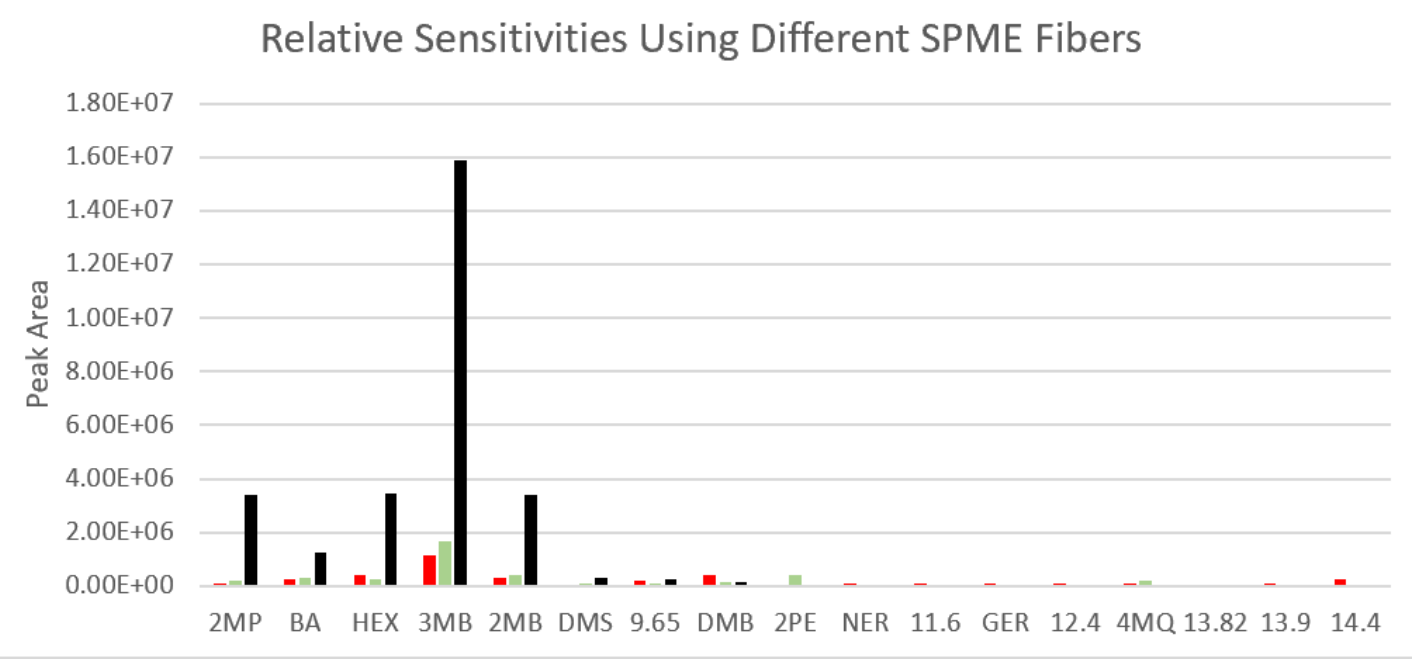

Sensitivities with Caboxen/PDMS Signals up to 6 Minutes

\section{Excluded}

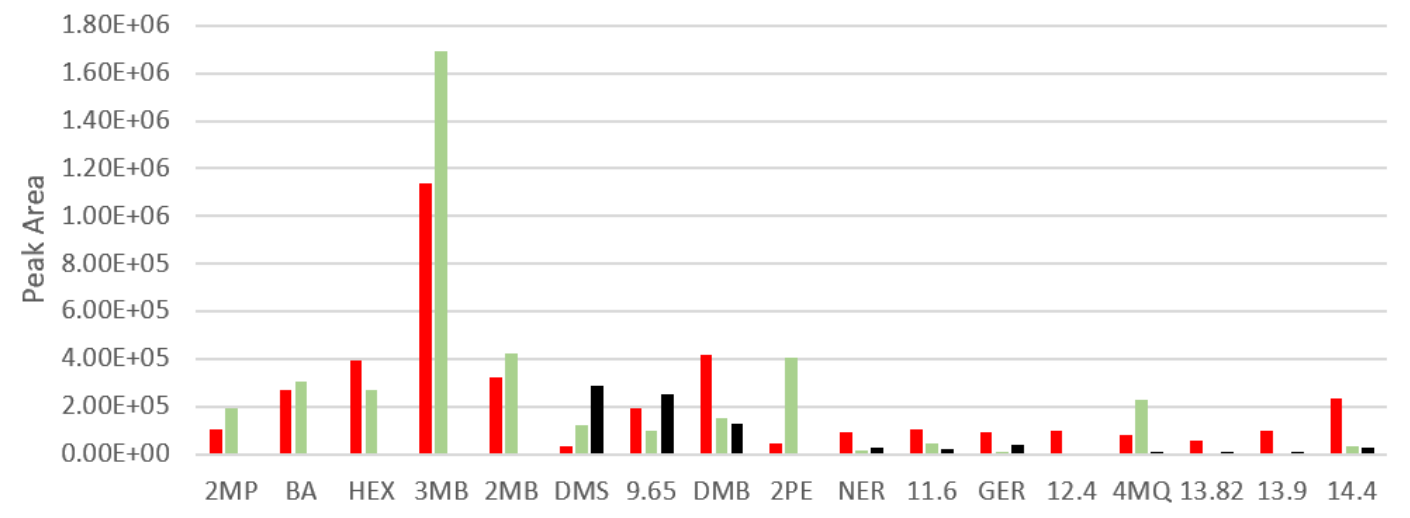

Peak Sensitivities Past 6 Minutes

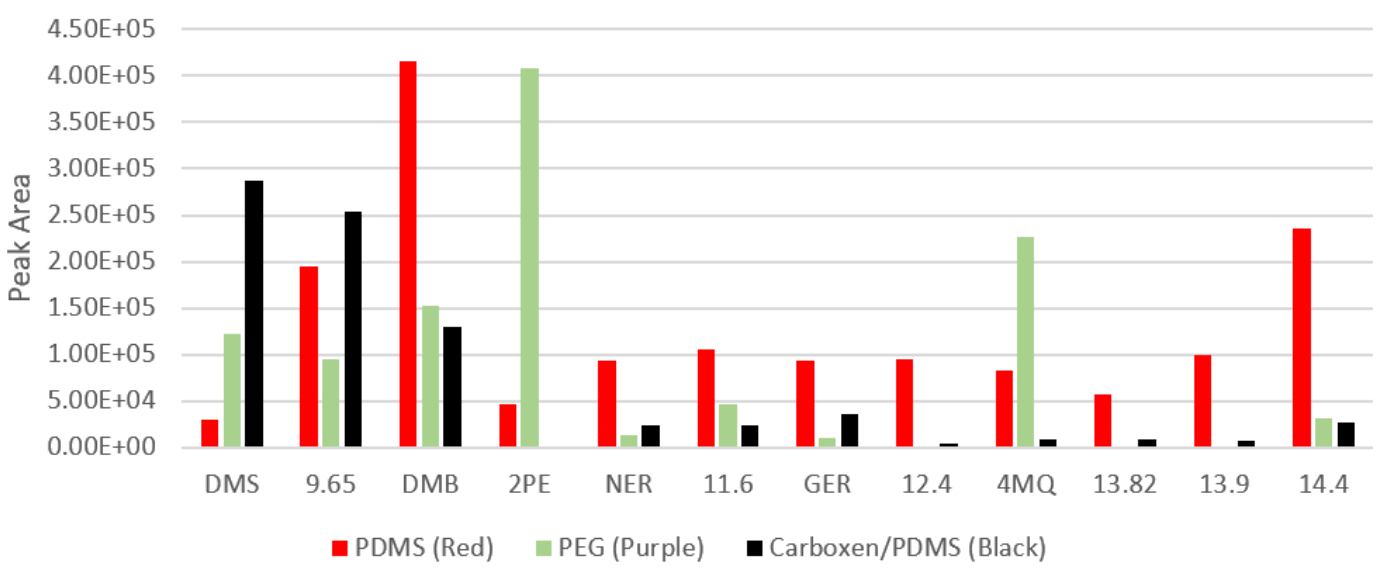

Figure S2. Relative Signals of Spider-Produced Volatile Compounds Extracted with Different SPME fibers. Signals of Known Compounds are Listed by Abbreviation; Signals of Unknown Compounds are Listed by Retention Time. 


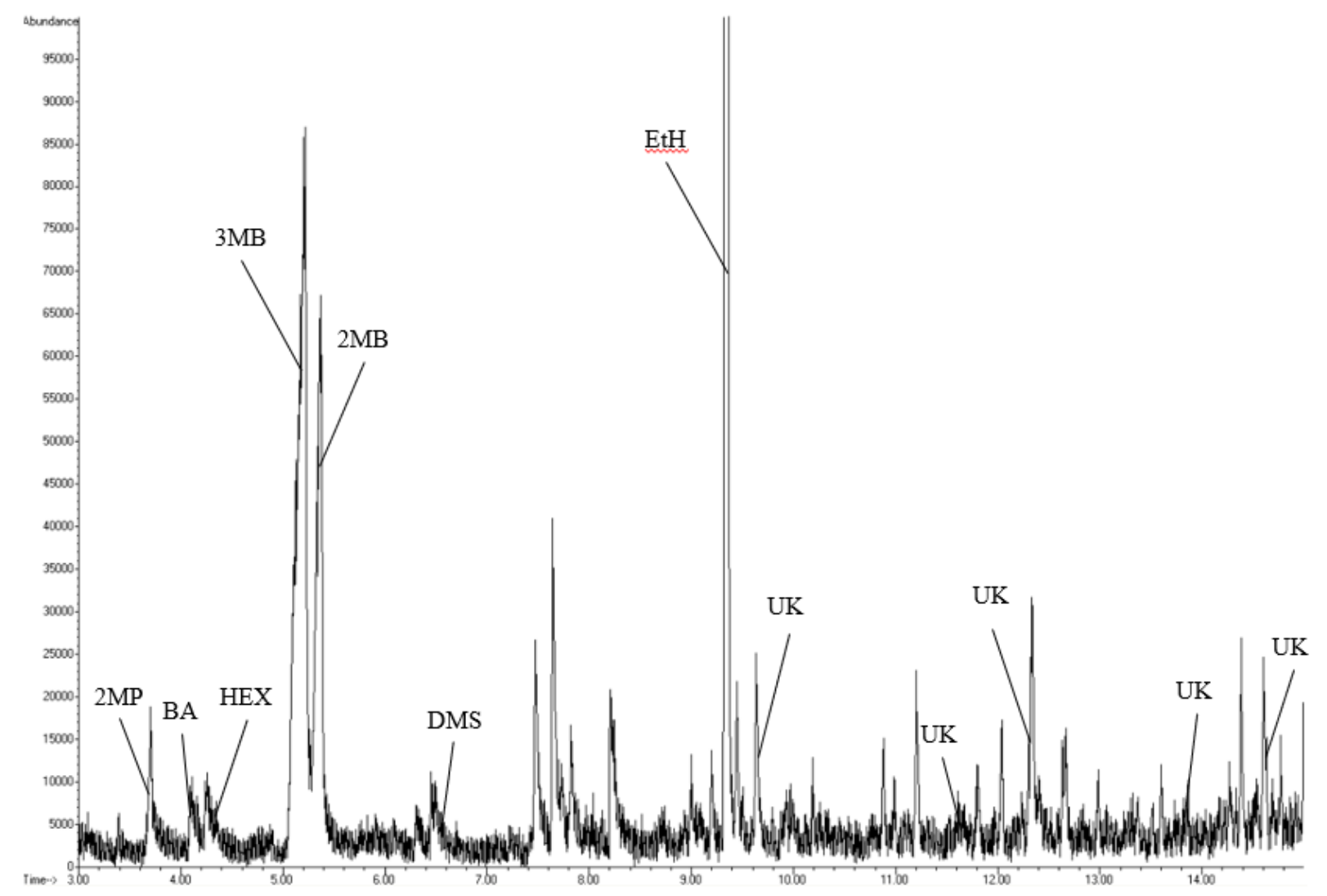

Figure S3. A representative chromatogram of Plectreurys tristis spider with Internal Standard. 